www.nature.com/hr

\title{
Do we understand the relationship between left ventricular structural remodeling and stroke in arterial hypertension?
}

\author{
Marijana Tadic and Cesare Cuspidi \\ Hypertension Research (2014) 37, 801-802; doi:10.1038/hr.2014.106; published online 19 June 2014
}

\section{LEFT VENTRICULAR HYPERTROPHY AND STROKE}

T eft ventricular hypertrophy (LVH) is a Lwell-known cardiovascular risk factor. Two decades ago, the results from the Framingham study revealed the relationship between LV mass and risk of stroke in the elderly. Verdecchia et al., ${ }^{1}$ in a population of 2363 initially untreated hypertensive patients, demonstrated that LVH significantly increased the risk of stroke and transient ischemic attack independent of blood pressure and other risk factors. The authors showed that LVH based on echocardiography increased the risk of cerebrovascular events by $64 \%$ in hypertensive patients (relative risk 1.64; 95\% confidence interval (CI): 1.07-2.68). Similar findings have been published more recently using a smaller group of hypertensive patients. ${ }^{2}$

Di Tullio et al. ${ }^{3}$ conducted a case-control study that included 394 patients with his or her first ischemic stroke and 413 age-, sexand race-ethnicity-matched controls, finding that LVH was associated with an increased stroke risk (adjusted odds ratio (OR) 2.5; 95\% CI: 1.7-3.5). This increase was observed in all age, sex and race-ethnic groups. Interestingly, the authors did not find a significant interaction between LVH and arterial hypertension and other risk factors on stroke risk. ${ }^{3}$

A recently published meta-analysis that included 12809 hypertensive patients from 14 different studies with a follow-up from 0.5 to 5 years demonstrated that the LV mass

M Tadic is at University Clinical Hospital Center 'Dr Dragisa Misovic_-Dedinje', Belgrade, Serbia; C Cuspidi is at Clinical Research Unit, University of Milan-Bicocca and Istituto Auxologico Italiano, Meda, Italy

E-mail: marijana_tadic@hotmail.com reduction induced by antihypertensive treatment significantly reduced the risk of stroke (OR 0.76, 95\% CI: 0.64-0.89, $P<0.001)$ but did not change the risk of myocardial infarction or heart failure. ${ }^{4}$ Verdecchia et al. ${ }^{1}$ previously published similar results in a population of 880 initially untreated hypertensive subjects.

In the last several years, a number of large studies have confirmed the relationship between LVH and cerebrovascular events in a general population. 5,6 The MESA study, which included 5098 participants initially free of cardiovascular disease, showed that LV mass adjusted for body size was positively associated with the incidence of stroke (hazard ratio (HR) 1.2 per $10 \%$ increment, $P=0.01) .{ }^{5}$ Bouzas-Mosquera et al. ${ }^{6}$ included 40138 adult patients from the general population (mean age $61.1 \pm 16.4$ ) and followed them for $5.6 \pm 3.9$ years. The investigators found that LV mass had a graded, independent association with allcause mortality, myocardial infarction and stroke (HR 1.26, 95\% CI: 1.13-1.40, $P<0.001$ in women and HR 1.19, 95\% CI: 1.09-1.30, $P<0.001$ in men). ${ }^{6}$ When the authors analyzed specific types of stroke separately, LV mass was significantly related to ischemic stroke in both genders and to hemorrhagic stroke in men, with a nonsignificant trend in women. ${ }^{6}$ Similar findings were shown in a small observational study in Sri Lanka in patients hospitalized for ischemic stroke.

In this issue of Hypertension Research, Wang et al. ${ }^{7}$ reports a significant relationship between $\mathrm{LVH}$ and stroke in a hypertensive Chinese population, and the results of this study largely confirm previous knowledge on this topic. The authors found that LVH was associated with an increased stroke risk (adjusted OR 1.52; 95\% CI: 1.25-1.85; multivariateadjusted OR, 1.43; 95\% CI: 1.16-1.75). This trend was observed in both genders equally, but not in different age groups. In particular, Wang et al. ${ }^{7}$ showed that LVH was not a risk factor for stroke between 40 and 55 years of age but was associated with an increased stroke risk in hypertensive patients between 55 and 74 years of age. This result is not in accordance with Di Tullio et al., ${ }^{3}$ who found this association in all age groups.

The investigators also revealed that the relative LV wall thickness is associated with an increased risk of stroke independent of LV mass index and other risk factors of stroke (adjusted OR 3.97; 95\% CI: 1.10-14.34). The authors used two different formulas to calculate the LV mass index and LV relative wall thickness, and succeeded in demonstrating a relationship between all four of these $\mathrm{LV}$ structural parameters and risk of stroke in a hypertensive population. ${ }^{7}$ This finding has important clinical implications because the authors utilized the two most frequently used formulas of LV mass index, adjusted by body surface area or body height, and the two most commonly used equations for LV relative wall thickness. The obtained results show that in everyday clinical practice, which formula a clinician uses is not important because the conclusion will remain the same.

\section{LEFT VENTRICULAR GEOMETRY AND STROKE}

The influence of LV geometry on stroke occurrence is less investigated. Di Tullio et $a .^{3}$ reported that concentric LVH carries 
the greatest stroke risk, followed by eccentric hypertrophy. The authors claimed that LV concentric remodeling was associated with a modest risk increase of stroke. The risk of stroke was increased independent of arterial hypertension and other risk factors. ${ }^{3}$ The researchers also found a relationship between LV geometry and type of stroke. Concentric LVH was more associated with lacunar (32.7\%) and cryptogenic (23.1\%) strokes than other LV geometries. Eccentric LVH was associated with cardioembolic stroke (26.5\%), whereas concentric remodeling was associated with lacunar stroke $(26.2 \%){ }^{3}$ Concentric LVH was revealed to be the most frequent type of LV geometry among patients with ischemic stroke.

The LIFE study showed that concentric remodeling (HR 2.99; 95\% CI: 1.16-7.71, $P<0.05$ ), eccentric hypertrophy (HR 1.79; 95\% CI: $1.17-2.73, P<0.05)$ and concentric hypertrophy (HR 2.71; 95\% CI: 1.13-6.45, $P<0.05)$ are independent predictors of primary composite end points (cardiovascular death, fatal or non-fatal stroke, and fatal or non-fatal myocardial infarction) ${ }^{8}$ Indeed, the composite end points in this study were cardiovascular death, myocardial infarction and stroke; thus, the results could not be the same as the studies that only investigated the relationship between LV geometry and stroke. ${ }^{3}$ However, this study also confirms the fact that LV geometry has an important influence on stroke occurrence.

The recently published AFFIRM trial showed that LV geometry does not affect stroke occurrence in hypertensive patients with atrial fibrillation, although concentric $\mathrm{LVH}$, unlike eccentric hypertrophy and concentric remodeling, increased mortality in these patients. ${ }^{9}$

In this issue, Wang et al. ${ }^{7}$ report that $\mathrm{LV}$ concentric hypertrophy carries the highest risk of stroke (unadjusted OR 1.93, 95\% CI: 1.48-2.53; adjusted OR 1.62, 95\% CI: $1.21-$ 2.17), followed by eccentric hypertrophy (unadjusted OR 1.61, 95\% CI: 1.08-2.54; adjusted OR 1.51, 95\% CI: 1.12-2.03), and concentric remodeling ranked third (unadjusted OR 1.47, 95\% CI: 1.12-1.93; adjusted OR 1.34, 95\% CI, 1.01-1.80). Concentric hypertrophy increased stroke risk in both genders in unadjusted and adjusted models. Eccentric hypertrophy was related to an increased stroke risk in both genders in unadjusted models, but was only associated with stroke risk in women in the adjusted model; in contrast, concentric remodeling was associated with stroke risk in women in all models.?

\section{POSSIBLE MECHANISMS LINKING LV REMODELING AND STROKE OCCURRENCE}

The particular mechanism explaining the relationship between $\mathrm{LVH}$ and increased risk of stroke is unclear, but there are several possibilities. First, LVH increases myocardial oxygen consumption, which can induce an imbalance between oxygen demand and supply in the myocardium, consequently leading to myocardial ischemia ${ }^{10}$ and inducing small areas of hypokinetic myocardium. These areas might serve as an origin for small thrombi to provoke an ischemic stroke. Second, LVH positively correlates with carotid intima-media thickness, which represents a significant predictor of stroke. In addition, hypertension is also an important predictor of carotid atherosclerosis, which significantly contributes to stroke occurrence. Third, LVH is associated with a higher prevalence of atrial fibrillation, which is a significant risk factor for stroke. Fourth, LVH is usually associated with other risk factors, such as obesity, insulin resistance, diabetes and metabolic syndrome, which significantly increase the risk of stroke. Fifth, a molecular mechanism could also explain the relationship between LVH and stroke; for example, a polymorphism of the platelet glycoprotein IIIa gene, present in high-risk hypertensive patients, increases the risk of ischemic stroke in these patients. ${ }^{11}$

The association between LV geometry and stroke is even less clear and more controversial. Studies have shown that asymptomatic lacunar lesions are more frequent in patients with an abnormal LV geometry. ${ }^{12}$ Arterial structure and function are abnormal in patients with concentric LVH and may partly explain the increased risk of stroke. Neurohormonal changes, such as aldosterone and atrial natriuretic peptide elevations, are related to an abnormal LV geometry (concentric and eccentric LV hypertrophy) $)^{13}$ and could partly be responsible for stroke occurrence. Di Tullio et al. ${ }^{3}$ showed that increased LV relative wall thickness was more frequently associated with lacunar infarcts, indicating an important role for LV relative wall thickness in the development of small-vessel disease, which could induce strokes. In the aforementioned study, cryptogenic stroke was less present in the patients with concentric $\mathrm{LVH} ;{ }^{3}$ however, eccentric LVH seems to be associated with cardioembolism, suggesting that LV dilation has an important role as a possible embolic source.
Further investigation is required to clarify the complex association between LV remodeling (LV mass and geometry) and stroke occurrence and possible approaches to reduce stroke risk.

\section{CONFLICT OF INTEREST}

The authors declare no conflict of interest.

1 Verdecchia P, Porcellati C, Reboldi G, Gattobigio R Borgioni C, Pearson TA, Ambrosio G. Left ventricular hypertrophy as an independent predictor of acute cerebrovascular events in essential hypertension. Circulation 2001; 104: 2039-2044.

2 Castilla-Guerra L, Fernández-Moreno M, Alvarez-Suero J, Gonzalez A. Echocardiographic assessment of hypertensive left ventricular hypertrophy in patients with acute ischemic stroke or transient ischemic attack. J Stroke Cerebrovasc Dis 2012; 21: 745-748.

3 Di Tullio MR, Zwas DR, Sacco RL, Sciacca RR, Homma S. Left ventricular mass and geometry and the risk of ischemic stroke. Stroke 2003; 34 2380-2384.

4 Costanzo P, Savarese G, Rosano G, Musella F, Casarett L, Vassallo E, Paolillo S, Marsico F, Rengo G, Leosco D, Perrone-Filardi P. Left ventricular hypertrophy reduction and clinical events. A meta-regression analysis of 14 studies in 12,809 hypertensive patients. Int $J$ Cardiol 2013; 167: 2757-2764.

5 Bluemke DA, Kronmal RA, Lima JA, Liu K, Olson J, Burke GL, Folsom AR. The relationship of left ventricular mass and geometry to incident cardiovascular events: the MESA (Multi-Ethnic Study of Atherosclerosis) study. J Am Coll Cardiol 2008; 52 : 2148-2155.

6 Bouzas-Mosquera A, Broullón FJ, Álvarez-García N, Peteiro J, Mosquera VX, Castro-Beiras A. Association of left ventricular mass with all-cause mortality, myocardial infarction and stroke. PLoS One 2012; 7 e45570.

7 Wang S, Xue $H$, Zou $Y$, Sun $K$, Fu C, Wang $H$, Hui R. Left ventricular hypertrophy, abnormal ventricular geometry and relative wall thickness are associated with increased risk of stroke in hypertensive patients among the Han Chinese. Hypertens Res 2014; 37 870-874.

8 Gerdts E, Cramariuc D, de Simone G, Wachtell K, Dahlöf B, Devereux RB. Impact of left ventricular geometry on prognosis in hypertensive patients with left ventricular hypertrophy (the LIFE study). Eur J Echocardiogr 2008; 9: 809-815.

9 Apostolakis S, Sullivan RM, Olshansky B, Lip GY. Left ventricular geometry and outcomes in patients with atrial fibrillation: the AFFIRM Trial. Int J Cardiol 2014; 170: 303-308.

10 Houghton JL, Frank MJ, Carr AA, von Dohlen TW, Prisant LM. Relations among impaired coronary flow reserve, left ventricular hypertrophy and thallium perfusion defects in hypertensive patients without obstructive coronary artery disease. J Am Coll Cardiol 1990; 15: 43-51.

11 Lanni F, Santulli G, Izzo R, Rubattu S, Zanda B, Volpe M, laccarino G, Trimarco B. The PI(A1/A2) polymorphism of glycoprotein IIla and cerebrovascular events in hypertension: increased risk of ischemic stroke in high-risk patients. J Hypertens 2007; 25 551-556.

12 Kohara K, Zhao B, Jiang Y, Takata Y, Fukuoka T, Igase M, Miki T, Hiwada K. Relation of left ventricular hypertrophy and geometry to asymptomatic cerebrovascular damage in essential hypertension. $A m ~ J$ Cardiol 1999; 83: 367-370.

13 Muscholl MW, Schunkert H, Muders F, Elsner D, Kuch B, Hense HW, Riegger GA. Neurohormonal activity and left ventricular geometry in patients with essential arterial hypertension. Am Heart J 1998; 135: 58-66 